\title{
A Petri Net Model for Part Sequencing and Robot Moves Sequence in a 2-Machine Robotic Cell
}

\author{
Mohammad Fathian ${ }^{1}$, Isa Nakhai Kamalabadi ${ }^{2}$, Mehdi Heydari ${ }^{1}$, Hiwa Farughi ${ }^{1}$ \\ ${ }^{1}$ Department of Industrial Engineering, Iran University of Science and Technology, Tehran, Iran; ${ }^{2}$ Department of Industrial Engi- \\ neering, Tarbiat Modares University, Tehran, Iran. \\ Email: h_farughi@iust.ac.ir
}

Received September $6^{\text {th }}, 2011$; revised October $10^{\text {th }}, 2011$; accepted October $22^{\text {nd }}, 2011$.

\begin{abstract}
This paper deals with part sequencing and optimal robot moves sequence in 2-machine robotic cells according to Petri net graph. We have assumed that the robotic cell is capable of producing same and different parts. We have considered a new motion cycle for robot moves sequence which is the development of existing motion cycles in 2-machine robotic cells. The main goal of this study is to minimize the cycle time by determining the optimal part sequencing and robot moves sequence in the robotic cell. So, we have proposed a model based on Petri network.
\end{abstract}

Keywords: Cycle Time, 2-Machine Robotic Cell, Petri Networks, Part Sequencing, Robot Moves Sequence

\section{Introduction}

In the present competitive world, time is an important and determining factor in industries. Along with technological progress in industries and organizations, managers's decision-making and their organizational activities and strategies have become increasingly complex. One of these strategies is the development of automation in industries and manufacturing organizations, which involves the use mechanical and programmable devices called robots for moving parts between the different stations. By establishing machines in cellular layout and using robots for automating the process, managers try to reduce the production time in order to increase the effectiveness of the production line, and to increase the productivity output in robotic manufacturing cells. In the last few years, researchers have been concerned with optimizing the robot move sequence in order to reduce production time in robotic manufacturing cells and many studies have been done in this regard.

The study of Sethi et al. [1] is considered as the beginning point of the robotic cell scheduling literature. They discussed on minimizing the cycle time in the single machine robotic cell. Sethi et al. [2] proved that in bufferless single-gripper two-machine robotic cells producing single part-type and having identical robot travel times between adjacent machines and identical load/unload times, a 1-unit cycle provides the minimum per unit cycle time in the class of all solutions, cyclic or otherwise.
For three machine case, Crama and van de Klundert [3], and Brauner and Finke [4] shown that the best 1-unit cycle is optimal solution for the class of all cyclic solutions. Hall et al. $[5,6]$ considered the computational complexity of the multiple-type parts three-machine robotic cell problem under various robot movement policies. I. N. Kamalabadi et al. [7] provided a new solution for the cyclic multiple parts three-machine robotic cell. They also $[8,9]$ considered the minimizing of cycle time in a blocking flow shop cell. This problem is studied for no-wait robotic cells too. For example Agnetis [10] found an optimal part schedule for no-wait robotic cells with three and two machines. Agnetis and pacciarelli [11] have studied part scheduling problem for no-wait robotic cells, and found the complexity of the problem. Crama et al. [3,12] studied flow-shop scheduling problems, models for such problems, and complexity of these problems. Dawande et al. [13] reviewed the recent developments in robotic cells and, provided a classification scheme for robotic cells scheduling problem. Some other special cases have been studied such as: Drobouchevitch et al. [14] provided a model for cyclic production in a dual-gripper robotic cell. Deineko et al. [15] studied the special case of two machine flexible robotic cell that the first machine performs one operation, and the second machine processes $\mathrm{K}$ operations step by step. Akturk et al. [16] studied on robotic cell scheduling with operational flexibility. Gultekin et al. [17] studied robotic cell scheduling problem with tooling 
constraints for a two-machine robotic cell where some operations can only be processed on the first machine and some others can only be processed on the second machine and the remaining can be processed on both machines. Gultekin et al. [18] considered a flexible manufacturing robotic cell with identical parts in which machines are able to do different operations and the operation time is not system parameter and is variable. They proposed a lower bound for 1-unit cycles and 2-unit cycles. Sriskandarajah et al. [19] classified the part sequence problems associated with different robot movement policies, in this paper a robot movement policy is considered, which its part scheduling problem is NPHard, and Baghchi et al. [20] proposed to solve this problem, by a heuristic or meta-heuristic.

It is obvious that the two fundamental problems in robotic cell scheduling are part sequencing and optimizing the robot move sequence. If the cell is meant to produce identical parts, the scheduling problem will depend on finding the optimal robot move sequence. In this paper, we have defined a new cycle for robot move sequence in a 2-machine robotic manufacturing cell, which is development of existing robot motion cycles. Our purpose is to obtain the optimal cycle time by determining the optimal parts entry to the cell. For the modeling this problem, timed Petri network has been used. In Section 2, assumptions, concepts and the robot move sequence for the proposed new cycle are introduced and the cycle time is calculated. In Section 3, concepts and relations in a Petri network are described. Then, the proposed motion cycle is described in full detail according to Petri net model. At first the mathematical model for the problem of producing identical parts in the production cycle is obtained, and then the model is generalized to the problem of producing different parts. Thereby, by determining the optimal part sequencing for the proposed cycle the minimum cycle time is obtained. In section 4 the results are analyzed and concluded.

\section{Problem Definition}

In robotic manufacturing cell scheduling, the major problem is how to determine the sequence of robot moves and order the parts entry in a cell that produces different parts. In past studies about 2-machine robotic cells, $S_{12} S_{21}, S_{2}, S_{1}$ motion cycles have been introduced [1], and the robot move sequence and the order of parts entry in 2-machine robotic cells have been studied [2]. In 3Machine robotic cells, the sequence of robot moves and parts entry to the cell has been investigated for both the same parts and different parts manufacturing cells [3,21]. In this paper, we have defined a new cycle for two-machine robotic cells and have considered the problem of different parts entry sequencing to obtain the optimum cycle time by a timed Petri net model. In most studies on scheduling 2-machine robotic cells the problem of flow shop has been considered. Thereby, each part is processed on the first machine and then conveyed by the robot to the second machine to be processed on. Indeed, each part must be processed on both machines. It is also assumed that the two machines are identical, i.e. the processing time of a same operation is the same on both machines. In addition, the robot's movement time between any two consecutive locations is the same and it is additive between different locations. Also, the robot's loading and unloading time in all conditions is the same and the robot has a linear movement in the cycle.

\section{Definition 1:}

Activity $\left(A_{i j}\right)$ signifies the robot's conveying a part from location $i$ to location $j$.

\section{Definition 2:}

A n-unit cycle means that the robot has entered $n$ parts into the cycle and for doing all the required processes on $\mathrm{n}$ parts each activity has been repeated $\mathrm{n}$ times. At the end of each cycle, $n$ part should be taken out of the cell by robot. Also, the beginning and the end mode of the n-unit cycle should be same.

\section{Definition 3:}

The n-unit cycle time is defined as the time needed to produce $\mathrm{n}$ parts in a cyclic process that a robot starts from the initial state and moves in a specific sequence, so that the necessary operations for producing $n$ parts is performed and then the robot goes on the initial position. Also, if we assume that the machines are flexible, in other words if each machine is capable of performing all operations on each part so that every part is processed completely by a single machine, then a new motion cycle can be defined with a sequence of moves as the following:

At the beginning of the cycle, a part is being processed by the second machine and the robot is located opposite the input area. According to definition 1 , the sequence of the robot movements is $A_{01} A_{23} A_{02} A_{13}$, i.e. the robot picks a part on the input buffer (the needed time is $\varepsilon$ ) and takes the part to the first machine $(\delta)$. Then it loads the part on the first machine $(\varepsilon)$ and moves towards the second machine $(\delta)$ and waits opposite this machine until it completes processing the part $\left(w_{2}\right)$, after the second machine has completed the process, the robot unloads the part from the second machine $(\varepsilon)$ and takes it to the output buffer $(\delta)$, then it loads it on the output buffer $(\varepsilon)$. Then the robot comes back to the input buffer (3 $\varepsilon)$, picks a part from the input buffer $(\varepsilon)$, moves it to the second machine $(2 \delta)$, and loads it on it $(\varepsilon)$, then it turns back to the first machine $(\delta)$ and waits opposite the machine until it completes processing the part $\left(w_{1}\right)$. 
When processing is finished, the robot unloads the part from the first machine $(\varepsilon)$, takes it to the output $(2 \delta)$ and then loads it on output area $(\varepsilon)$. Then it comes back to the input buffer $(3 \delta)$. Thereby, the cycle is finished and the robot returns to the initial position. During this cycle, two parts are produced, so the cycle is called 2-unit cycle. In this cycle, it is assumed that the robot has no waiting time in the input and the output buffer. It should be noted that since each machine has the ability to perform all operations, in the process of producing $n$ parts and in each sequence of cyclic moves, after one stage we have a repetitive sequences of robot movements which continues until $\mathrm{n}$ parts are produced. In this proposed cycle, the starting point of the mentioned repetitive process is the beginning of the cycle.

In this case, the cycle time for producing two parts is calculated as follows, and its parameters are:

$\varepsilon$ : Loading or unloading time

$\delta$ : The time in which the robot moves between two successive locations

P: Processing time of each part on the machines

$w_{i}$ : Waiting time in front of machine $i$

$\mathrm{C}_{\mathrm{t}}$ : Cycle time:

$$
\begin{aligned}
2-\text { unit } C_{t}= & \varepsilon+\delta+\varepsilon+\delta+w_{2}+\varepsilon+\delta+\varepsilon+3 \delta+\varepsilon+2 \delta+\varepsilon \\
& +\delta+w_{1}+\varepsilon+2 \delta+\varepsilon+3 \delta=8 \varepsilon+14 \delta+w_{1}+w_{2}
\end{aligned}
$$

$$
\begin{aligned}
& w_{1}=\operatorname{Max}\left\{0, P-\left(8 \varepsilon+4 \delta+w_{2}\right)\right\} \\
& w_{2}=\operatorname{Max}\{0, P-(2 \varepsilon+4 \delta)\}
\end{aligned}
$$

Therefore, the time needed to produce a part in this cycle is:

$$
\begin{aligned}
1-\text { unit } C_{t}= & \frac{8 \varepsilon+14 \delta+w_{1}+w_{2}}{2} \\
1-\text { unit } C_{t}= & 4 \delta+7 \delta+\frac{1}{2} \operatorname{Max}\{0, P-(2 \varepsilon+4 \delta)\} \\
& +\frac{1}{2} \operatorname{Max}\left\{0, P-\left(4 \varepsilon+8 \delta+w_{2}\right)\right\}
\end{aligned}
$$

The layout of the proposed cycle and the initial position are shown in Figure 1.

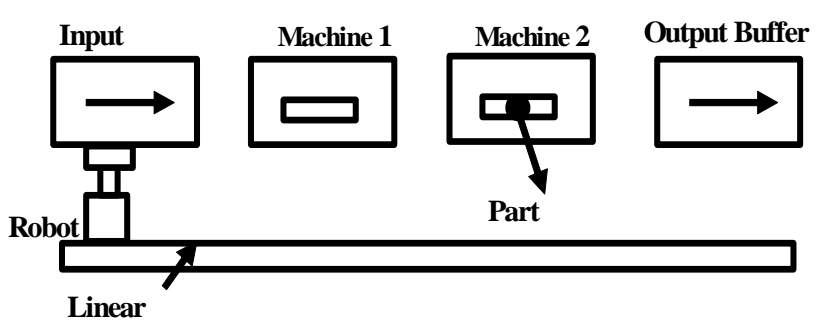

Figure 1. Layout of the new cycle.

\section{Petri Network Model}

Many systems can be modeled on Petri nets and it is possible to show their features using these networks [22]. Petri network is a suitable device for mathematical and graphical modeling. The graphical behavior of variables and the ability to convert them into flowchart and diagram is one of the important features of Petri nets [8]. Petri networks were introduced for the first time by Adam Petri in 1962. Lee and Yung (1955) presented a new method for planning flexible process sequences using Petri networks [22]. Petri nets are directed split graphs which are divided into two groups of place and transition. The directed arcs link some places to some transitions or link some transitions to some places. Notation in a Petri network is a vector whose elements show the number of arcs. Each Petri network is shown as $P N=\left\{P, T, A, W, M_{0}\right\} \quad$ [22] in which:

P: a finite set of places $P=\left\{P_{1}, P_{2} \ldots, P_{n}\right\}$

T: a finite set of transitions $t=\left\{t_{1}, t_{2} \ldots ., t_{m}\right\}$

A: a finite set of arcs $A \subset\{P \times T\} \cup\{T \times P\}$

$\mathrm{W}$ : weight function associated with each location $P=\{1,2, \ldots .\} W:$.

M0: Initial network markup $P=\{0,1,2, \ldots ..\} M_{0}: P$

Mark changes in a Petri network involving firing (transposition) is as the following:

1) The firing is performed when the location $P_{i}$ has at least $W\left(P_{i}, t\right)$ token, whereby $W\left(P_{i}, t\right)$ is the arc weight of Pi to t.

2) An active transposition may lead to firing depending on whether its movement is performed or not.

3) If the transition $\mathrm{t}$ is fired, $W\left(P_{i}, t\right)$ of tokens is reduced from each $P_{i}$ to $t$ input place and $W\left(P_{0}, t\right)$ number of tokens is added to each $\mathrm{P} 0$ output location of transition $\mathrm{t}$. are added. $\mathrm{W}\left(\mathrm{t}, P_{0}\right)$ is the arc weight of $\mathrm{t}$ to $P_{0}$ [22].

\section{Theorem 1:}

In a marked graph, for each location that has $M_{i}$ tokens the following relationship $S_{B} \geq S_{A}+M_{i} C_{t}$, whereby $S_{B}, S_{A}$ are the starting times of transitions of $\mathrm{B}$ and $\mathrm{A}$, and $C_{t}$ is the cycle time in timed Petri network. Figure $\mathbf{2}$ shows this marked graph.

\subsection{Timed Petri Network Model for Producing Identical Parts in the New Cycle}

At the beginning of the proposed cycle, it is assumed that a part on the second machine is being processed and the

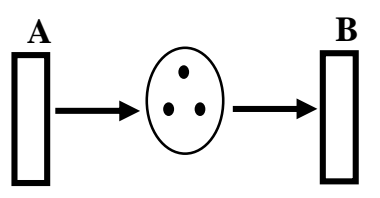

Figure 2. Marked graph related to theorem 1. 
robot is located opposite the input buffer. For simplicity of calculation and drawing Petri networks, in modeling the problem and drawing its graph we assume that the starting point of the cycle moves to the moment when the robot loads the first machine and leaves for the second machine; at this moment a part is being processed by both machines. At the end of the cycle the robot returns to this situation. Accordingly, the graph for the same parts production cycle is shown in "Figure 3".

The parameters needed for modeling this problem by Petri network are the following:

$R_{1}$ : moving toward the second machine and waiting opposite the second machine

\section{$\left(\delta+w_{2}\right)$}

$R_{2}$ : unloading the part from the second machine ( $\varepsilon$ )

$R_{3}$ : moving towards the output buffer, loading the part on the output buffer, moving towards the input buffer, unloading the part from the input buffer and moving towards second machine ( $6 \delta+2 \varepsilon$ )

$R_{4}$ : loading the part on the second machine ( $\varepsilon$ )

$R_{5}$ : moving toward the first machine and waiting opposite the first machine $\left(\delta+W_{1}\right)$

$R_{6}$ : the robot's unloading the part on the first machine $(\varepsilon)$

$R_{7}$ : moving towards the output buffer and loading the part on the output buffer, moving towards the input buffer, unload the part from the input buffer, and moving toward the first machine $(6 \delta+2 \varepsilon)$

$R_{8}$ : loading the part on the first machine ( $\varepsilon$ ) $M_{1}$

$\alpha^{\prime}$ : starting moment of the first machine's processing

$\alpha$ : The moment that the first machine has finished its

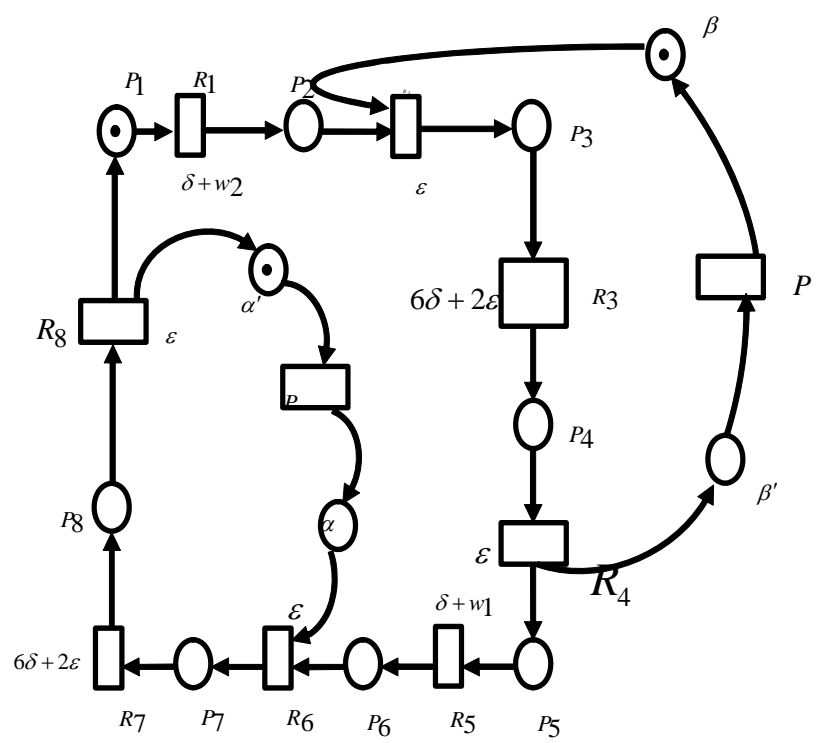

Figure 3. Petri network graph for same parts production cycle. task and is waiting for the robot to unload the part from it

$\beta^{\prime}$ : starting moment of the second machine's processing $\left(M_{2}\right)$

$\beta$ : The moment that the second machine has finished its task and is waiting for the robot to unload the part from it

$P$ : operating time of the first or the second machine

Mathematical model for production planning problem of producing same parts in the proposed cycle are the following:

Min $\mathrm{C}_{\mathrm{t}}$

Subject to:

$$
\begin{aligned}
& P_{1}: S_{1}-S_{8}+C_{t}=\varepsilon \\
& P_{2}: S_{2}-S_{1}=\delta+w_{2} \\
& P_{3}: S_{3}-S_{2}=\varepsilon \\
& P_{4}: S_{4}-S_{3}=6 \delta+2 \varepsilon \\
& P_{5}: S_{5}-S_{4}=\varepsilon \\
& P_{6}: S_{6}-S_{5}=\delta+w_{1} \\
& P_{7}: S_{7}-S_{6}=\varepsilon \\
& P_{8}: S_{8}-S_{7}=6 \delta+2 \varepsilon \\
& \alpha: S_{6}-S_{8}+C_{t} \geq \varepsilon+P \\
& \beta: S_{2}-S_{4}+C_{t} \geq P+\varepsilon \\
& \quad S_{i}, w_{1}, w_{2} \geq 0
\end{aligned}
$$

In this model, the objective function is to minimize the cycle time of producing same parts. The constraints are written according to the properties of timed Petri network and what were written in "Theorem 1".

\subsection{Mathematical Model for Production Planning Problem of Producing Different Parts in the Proposed Cycle}

In the proposed new cycle, we assume that $\mathrm{n}$ different parts must be produced, and that the processing time for all parts is specified. Therefore, we can model the Petri network in a way that the optimal sequence of parts' entering the cell is determined. According to this model, $n$ parts are produced by this production cycle in the minimum possible time. Petri network graph related to the production of these $\mathrm{n}$ parts is obtained by repeating the same parts production cycle $n / 2$ times as described in the previous section. Because in the proposed same parts production cycle 2 parts are produced, for producing $n$ parts in the cycle, the mentioned sequence must be repeated $n / 2$ times. For modeling the new proposed different parts production cycle, in addition to the parameters used for producing identical parts, the following parameters are needed: 
$X_{1 i j}$ if the part $\mathrm{i}$, is the $j^{\text {th }}$ part given to the first machine $X_{2 i j}$ : if the part $\mathrm{i}$, is the $j^{\text {th }}$ part given to the second machine

t: part counter $t \neq i$

Mathematical model for production planning problem with different parts are the following:

$\min C_{t}$

Subject to:

$$
\begin{aligned}
& P_{1,1}: S_{1,1}-S_{8, n}+C_{t}=\varepsilon \\
& P_{1, j}: S_{1, j}-S_{8, j}=\varepsilon \quad j=2, \ldots ., n \\
& P_{2, j}: S_{2, j}-S_{1, j}=\delta+w_{2} \quad j=1, \ldots ., n \\
& P_{3, j}: S_{3, j}-S_{2, j}=\varepsilon \quad j=1, \ldots ., n \\
& P_{4, j}: S_{4, j}-S_{3, j}=6 \delta+2 \varepsilon \quad j=1, \ldots ., n \\
& P_{5, j}: S_{5, j}-S_{4, j}=\varepsilon \quad j=1, \ldots ., n \\
& P_{6, j}: S_{6, j}-S_{5, j}=\delta+w_{1} \quad j=1, \ldots ., n \\
& P_{7, j}: S_{7, j}-S_{6, j}=\varepsilon \quad j=1, \ldots ., n \\
& P_{8, j}: S_{8, j}-S_{7, j}=6 \delta+2 \varepsilon \quad j=1, \ldots ., n \\
& \alpha_{1}: S_{6,1}-S_{8,1}+C_{t} \geq \sum_{i=1}^{n} x_{1 i n} \cdot a_{i}+\varepsilon \\
& \alpha_{j}: S_{6, j}-S_{8, j} \geq \sum_{i=1}^{n} x_{1 i j} \cdot a_{i}+\varepsilon \quad j=2, \ldots . ., n \\
& \beta_{1}: S_{2, n}-S_{4, n}+C_{t} \geq \sum_{i=1}^{n} X_{2 i n} \cdot b i+\varepsilon \\
& \beta_{j}: S_{2, j}-S_{4, j} \geq \sum_{i=1}^{n} X_{2 i j} \cdot b i+\varepsilon \quad j=1, \ldots ., n-1 \\
& \sum_{i=1}^{n} X_{1 i j}+\sum_{i=1}^{n} X_{2 i j}=1 \quad \forall j=1, \ldots \ldots, n \\
& \sum_{j=1}^{n} X_{1 i j}+\sum_{j=1}^{n} X_{2 i j}=1 \quad i=1, \ldots \ldots, n \\
& X_{2 i n}=X_{2 i 0}=1 \quad i=1, \ldots \ldots, n \\
& X_{1 i n+1}=X_{1 i 1}=1 \quad i=1, \ldots \ldots, n \\
& X 1_{i, j-1}\left(\sum_{t \neq i} X 2_{t, j}\right)+X 2_{i, j-1}\left(\sum_{t \neq i} X 1_{t, j}\right)=1 \forall i, t, j \\
& S_{i j}, w_{1}, w_{2} \geq 0 \\
& X_{1 i j}, X_{2 j j}=\{0,1\}
\end{aligned}
$$

Constraints added to this model are the following:

Constraint (32) states that at any stage only one part must enter and it must be assigned only to one machine.

Constraint (33) states that part i must enter only in one stage of the cycle.
Constraint (34) states that the part being processed on the second machine at the beginning of cycle is the $n^{\text {th }}$ part in the previous cycle and represents the sequence in new cycle.

Constraint (35) states that the part being processed by the first machine at the beginning of cycle is $n+1^{\text {st }}$ part in the previous production cycle.

Constraints (36) state that the sequence of parts entering the new cycle must be observed, i.e. if in a stage one part is given to one of the machines, in next stage the next part is given to the other machine.

Constraints $P_{i, j}$ are written according to "Theorem 1". Also, the objective function of the problem is to minimize the cycle time to produce $\mathrm{n}$ various parts in the new proposed cycle.

\section{Conclusions}

In this paper, at first the existing feasible robot moves cycles in a 2-machine robotic cells reviewed, then a new cycle with assumption that the machines are identical and flexible with the ability to perform all the necessary operations for producing the same and different parts, was introduced. The main problem in this research was to minimize the cycle time in producing same and different parts by optimizing part sequencing in the robotic cell. Accordingly, we provided a mathematical programming model based on Petri network. Among the issues that can be considered in future researches are: 1) to provide methods for solving the problem model and comparing the results; 2) to test the performance of the existing robot move sequences in the form of new proposed cycle; 3 ) to extend these problem to 3-machine robotic cells.

\section{REFERENCES}

[1] S. P. Sethi and D. Groupe, "D’Études et de Recherche en Analyse des, Sequencing of Robot Moves and Multiple Parts in a Robotic Cell,” Groupe D'Études et de Recherche en Analyse des Decisions, Montréal, 1989.

[2] S. P. Sethi, C. Sriskandarajah, G. Sorger, J. Blazewicz and W. Kubiak, "Sequencing of Parts and Robot Moves in a Robotic Cell," International Journal of Flexible Manufacturing Systems, Vol. 4, 1992, pp. 331-358. doi:10.1007/BF01324886

[3] Y. Crama and V. D. Klundert, "Cyclic Scheduling in 3-Machine Robotic Flow Shops," Journal of Scheduling, Vol. 2, 1999, pp. 35-54. doi:10.1002/(SICI)1099-1425(199901/02)2:1<35::AID-J OS15>3.0.CO;2-J

[4] N. Brauner and G. Finke, “On a Conjecture About Robotic Cells: New Simplified Proof for the Three machine Case,” INFOR, Vol. 37, No. 1, 1999, pp. 20-36

[5] N. G. Hall, H. Kamoun and C. Sriskandarajah, "Scheduling in Robotic Cells: Complexity and Steady State Analysis," European Journal of Operational Research, Vol. 
109, 1998, pp. 43-65. doi:10.1016/S0377-2217(96)00333-5

[6] N. G. Hall, H. Kamoun and C. Sriskandarajah, "Scheduling in Robotic Cells: Classification, Two and Three Machine Cells,” Operations Research, Vol. 45, 1997, pp. 421-439. doi:10.1287/opre.45.3.421

[7] I. N. Kamalabadi, S. Gholami and A. H. Mirzaei, "A New Solution for the Cyclic Multiple-Part Type Three-Machine Robotic Cell Problem Based on the Particle Swarm Meta-Heuristic," Journal of Industrial and Systems Engineering, Vol. 1, No. 4, 2008, pp. 304-317.

[8] I. N. Kamalabadi, "A New Formulation for Scheduling Problems Through Petri-nets,” In The Iranian Mathematical Conference, Iran, 1996.

[9] I. N. Kamalabadi, N. G. Hall and H. Sriskandarajah, "Minimizing Cycle Time in a blocking Flowshop," Operations Research, Vol. 48, 2000, pp. 177-180. doi:10.1287/opre.48.1.177.12451

[10] A. Agnetis, "Scheduling No-Wait Robotic Cells with Two and Three Machines," European Journal of Operational Research, Vol. 123, 2000, pp. 303-314. doi:10.1016/S0377-2217(99)00258-1

[11] A. Agnetis and D. Pacciarelli, "Part Sequencing in ThreeMachine No-Wait Robotic Cells,” Operations Research Letters, Vol. 27, 2000, pp. 185-192. doi:10.1016/S0167-6377(00)00046-8

[12] Y. Crama, V. Kats and V. D. Klundert, "Cyclic Scheduling in Robotic Flow Shops," Annals of Operation Research: Mathematics of Industrial Systems, Vol. 96, 2000, pp. 97-124.

[13] M. Dawande, H. N. Geismar, S. P. Sethi and C. Sriskandarajah, "Sequencing and Scheduling in Robotic Cells: Recent Developments,” Journal of Scheduling, Vol. 8, 2005, pp. 387-426. doi:10.1007/s10951-005-2861-9

[14] I. G. Drobouchevitch, S. P. Sethi and C. Sriskandarajah, "Scheduling Dual Gripper Robotic Cell One Unit Cycles," European Journal of Operational Research, Vol.
171, 2006, pp. 598-631. doi:10.1016/j.ejor.2004.09.019

[15] V. Deineko and G. Steiner, "Robotic-Cell Scheduling: Special Polynomially Solvable Cases of the Traveling Salesman Problem on Permuted Monge Matrices,” Journal of Combinatorial Optimization, Vol. 9, No. 4, 2005, pp. 381-399. doi:10.1007/s10878-005-1778-8

[16] M. S. Akturk, H. Gultekin and O. E. Karasan, "Robotic Cell Scheduling with Operational Flexibility," Discrete Applied Mathematics, Vol. 145, 2000, pp. 334-348. doi:10.1016/j.dam.2004.02.012

[17] H. Gultekin, M. S. Akturk and O. E. Karasan, "Cyclic Scheduling of a 2-Machine Robotic Cell with Tooling Constraints,” European Journal of Operational Research, Vol. 174, 2006, pp. 777-796. doi:10.1016/j.ejor.2005.03.021

[18] H. Gultekin, M. S. Akturk and O. E. Karasan, "Scheduling in a Three-Machine Robotic Flexible Manufacturing Cell,” Computers \& Operations Research, Vol. 34, 2007, pp. 2463-2477. doi:10.1016/j.cor.2005.09.015

[19] C. Sriskandarajah, N. G. Hall, H. Kamoun and H. Wan, "Scheduling Large Robotic Cells without Buffers," Annals of Operations Research, Vol. 76, 1998, pp. 287-321. doi:10.1023/A:1018952722784

[20] T. P. Bagchi, J. N. D. Gupta and C. Sriskandarajah, “A Review of TSP Based Approaches for Flow shop Scheduling,” European Journal of Operational Research, Vol. 169, 2006, pp. 816-854. doi:10.1016/j.ejor.2004.06.040

[21] Y. Crama, "Combinatorial Optimization Models for Production Scheduling in Automated Manufacturing Systems,” European Journal of Operational Research, Vol. 99, 1997, pp. 136-153. doi:10.1016/S0377-2217(96)00388-8

[22] J. Maggot, "Performance Evaluation of Concurrent Systems Using Petri Nets,” Information Processing Letters, Vol. 8, No. 1, 1984, pp. 7-13. doi:10.1016/0020-0190(84)90067-X 